Article

\title{
New Refinements for the Error Function with Applications in Diffusion Theory
}

\author{
Gabriel Bercu (D) \\ Department of Mathematics and Computer Sciences, “Dunărea de Jos" University of Galaţi, \\ 111 Domnească Street, 800201 Galaţi, Romania; Gabriel.Bercu@ugal.ro
}

Received: 19 November 2020; Accepted: 3 December 2020; Published: 6 December 2020

\begin{abstract}
In this paper we provide approximations for the error function using the Padé approximation method and the Fourier series method. These approximations have simple forms and acceptable bounds for the absolute error. Then we use them in diffusion theory.
\end{abstract}

Keywords: error function; rational refinement; Padé approximant; bound; Fourier series; analytic inequalities; even functions

\section{Introduction and Motivation}

The error function erf is a special function. It is widely used in many areas of research, such as: statistical computations, mathematical models in biology, mathematical physics, and diffusion theory.

In this work we intend to draw attention to the Padé approximant method and to the Fourier series method by some inequalities and approximations which involve the even function $\exp \left(-x^{2}\right)$ and the error function. the form

The error function is related to the function expression for a Gaussian distribution and has

$$
\operatorname{erf}(x)=\frac{2}{\sqrt{\pi}} \int_{0}^{x} e^{-u^{2}} d u .
$$

The complementary error function is

$$
\operatorname{erfc}(x)=1-\operatorname{erf}(x)
$$

or

$$
\operatorname{erfc}(x)=\frac{2}{\sqrt{\pi}} \int_{x}^{\infty} e^{-u^{2}} d u .
$$

The integral in the error function cannot be evaluated analytically, so values for $\operatorname{erf}$ and $\operatorname{erf} c$ are computed via approximations and often available in tables.

There are two main aspects in the study of the error function. The first aspect refers to the establishment of some bounds for the error function. The second aspect is to approximate the error function with different elementary functions such that the absolute error function is as small as possible.

The bounds for the error function have attracted the attention of many researches, since there were studies in different forms in the recent past (see [1-20]). 
Pólya [8] proved that the inequality

$$
\operatorname{erf}(x)<\sqrt{1-\exp \left(-\frac{4 x^{2}}{\pi}\right)}
$$

holds for all $x>0$.

Neumann [15] proved that the double inequality

$$
\frac{2 x}{\sqrt{\pi}} \cdot e^{-\frac{x^{2}}{3}} \leq \operatorname{erf}(x) \leq \frac{2 x}{\sqrt{\pi}} \cdot \frac{e^{-x^{2}}+2}{3}
$$

holds for all $x>0$.

Recently, Yang and Chu [13] improved the above inequalities by the following inequalities

$$
\begin{aligned}
\frac{2 x}{\sqrt{\pi}} \cdot e^{-\frac{x^{2}}{3}} & <\frac{2 x}{\sqrt{\pi}} \cdot \frac{e^{-\frac{x^{2}}{3}}+5 e^{-\frac{x^{2}}{5}}}{6}<\operatorname{erf}(x)<\frac{2 x}{\sqrt{\pi}} \cdot \frac{5 e^{-\frac{3 x^{2}}{5}}+4}{9}< \\
& <\frac{2 x}{\sqrt{\pi}} \cdot \frac{16 e^{-\frac{x^{2}}{2}}+5 e^{\frac{x^{2}}{5}}}{21}<\frac{2 x}{\sqrt{\pi}} \cdot \frac{20 e^{-\frac{2 x^{2}}{5}}+e^{x^{2}}}{21}
\end{aligned}
$$

for all $x>0$.

Komatsu's inequalities [16] assert that

$$
\frac{2 e^{-\frac{x^{2}}{2}}}{x+\sqrt{x^{2}+4}}<\int_{x}^{\infty} e^{-\frac{t^{2}}{2}} d t<\frac{2 e^{-\frac{x^{2}}{2}}}{x+\sqrt{x^{2}+2}}
$$

holds for all $x>0$.

Hastings [5] suggested some expressions, of which the simplest are

$$
\operatorname{erf}(x) \simeq 1-\left(a_{1} t+a_{2} t^{2}+a_{3} t^{3}\right) e^{-x^{2}}, 0 \leq x
$$

where

$$
t=\frac{1}{1+a_{4} x}, a_{1}=0.3480242, a_{2}=-0.0958798, a_{3}=0.7478556, a_{4}=0.47047
$$

and

$$
\operatorname{erf}(x) \simeq 1-\left(1+a_{1} x+a_{2} x^{2}+a_{3} x^{3}+a_{4} x^{4}\right)^{-4}
$$

where

$$
a_{1}=0.278393, a_{2}=0.230389, a_{3}=0.000972, a_{4}=0.078108 .
$$

The absolute error $\varepsilon(x)$ is shown to be less $2 \times 10^{-5}$ and $5 \times 10^{-4}$, respectively.

The approximation

$$
\operatorname{erf}(x) \simeq 1-2\left(1+\left(a_{1}+a_{2} x\right)^{a_{3}}\right)^{a_{4}}
$$

was obtained by Burr [2], where $a_{1}=0.644693, a_{2}=0.22908, a_{3}=4.874, a_{4}=-6.158$.

Norton [12] proposed the following approximation

$$
\operatorname{erf}(x) \simeq\left\{\begin{array}{c}
1-\exp \left(-\frac{2 x^{2}+1.2(x \sqrt{2})^{0.8}}{2}\right) ; 0 \leq x \leq 2.7 \\
-1+\sqrt{\frac{2}{\pi}} \exp \left(-x^{2}\right) ; x>2.7
\end{array}\right.
$$

with absolute error $|\varepsilon(x)|<8.07 \times 10^{-3}$, for all $x \geq 0$. 
Furthermore, in sigmoid functions theory, the function $\frac{\sqrt{\pi}}{2} \operatorname{erf}(x)$ is compared with the following algebraic functions

$$
\eta_{1}(x)=\frac{x}{1+|x|} \text { and } \eta_{2}(x)=\frac{x}{\sqrt{1+x^{2}}} .
$$

The aim of our work is to refine these inequalities. First, we will use the Padé approximant method in order to obtain a new approximation of the error function. The proposed approximation has a simple rational form and it is easy to programme.

It is known that a Padé approximant is the 'best' approximation of a function by a rational function of given order. The Padé approximant $[L, M]$ corresponds to the Taylor series. When it exists, the $[L, M]$ Padé approximant to any power series $A(x)=\sum_{j=0}^{\infty} a_{j} x^{j}$ is unique

The coefficients are found by setting

$$
A(x)=\frac{p_{0}+p_{1} x+\ldots+p_{L} x^{L}}{1+q_{1} x+\ldots+q_{M} x^{M}}
$$

These give the set of equations

$$
\left\{\begin{array}{l}
p_{0}=a_{0} \\
p_{1}=a_{0} q_{1}+a_{1} \\
p_{2}=a_{0} q_{2}+a_{1} q_{1}+a_{2} \\
\vdots \\
p_{L}=a_{0} q_{L}+\ldots+a_{L-1} q_{1}+a_{L} \\
0=a_{L-M+1} q_{M}+\ldots+a_{L} q_{1}+a_{L+1} \\
0=a_{L} q_{M}+\ldots+a_{L+M-1} q_{1}+a_{L+M}
\end{array}\right.
$$

For example, the first orders of Padé approximant for $\frac{\sqrt{\pi}}{2} \operatorname{erf}(x)$ are

$$
\begin{aligned}
& \text { Pade }[3,4]=A(x)=\frac{110 x^{3}+210 x}{39 x^{4}+180 x^{2}+210}, \\
& \text { Pade }[1,8]=B(x)=\frac{113,400 x}{29 x^{8}-660 x^{6}+1260 x^{4}+378,00 x^{2}+113,400}, \\
& \text { Pade }[5,6]=C(x)=\frac{302,379 x^{5}+1,027,320 x^{3}+10,242,540 x}{52,595 x^{6}+758,625 x^{4}+4,441,500 x^{2}+10,242,540} .
\end{aligned}
$$

We notice that the expressions $A(x), B(x), C(x)$ are positive for all $x>0$.

After several attempts we obtain that the best approximation for the function $\frac{\sqrt{\pi}}{2} \operatorname{erf}(x)$ is provided by Pade [5, 6] approximation.

The second idea is that the function $\exp \left(-x^{2}\right)$ is even, so it can be expanded in Fourier trigonometric series, e.g.,

$$
\begin{gathered}
\exp \left(-x^{2}\right)=1-a-b+a \cos x+b \cos 2 x+\ldots, \\
\exp \left(-x^{2}\right)=1-a-b-c+a \cos x+b \cos 2 x+c \cos 3 x+\ldots .
\end{gathered}
$$

In the following we will present our algorithm for the first expansion. We define the function $F_{1}(x)$ by

$$
F_{1}(x)=1-a-b+a \cos x+b \cos 2 x .
$$


The power series expansion of $\exp \left(-x^{2}\right)-F_{1}(x)$ near 0 is

$$
\frac{1}{2} x^{2}(a+4 b-2)+\frac{1}{24} x^{4}(-a-16 b+12)+O\left(x^{6}\right) .
$$

In order to increase the speed of the function $F_{1}(x)$ approximating $\exp \left(-x^{2}\right)$, we vanish the first coefficients as follows:

$$
\left\{\begin{array}{c}
a+4 b-2=0 \\
-a-16 b+12=0
\end{array}\right.
$$

and we obtain $a=-\frac{4}{3}$ and $b=\frac{5}{6}$.

Therefore we have

$$
\exp \left(-x^{2}\right)-\frac{3}{2}+\frac{4}{3} \cos x-\frac{5}{6} \cos 2 x=-\frac{17}{180} x^{6}+\frac{367}{10,080} x^{8}-\frac{2449}{302,400} x^{10} .
$$

Using the same algorithm, we find

$$
\exp \left(-x^{2}\right)+\frac{7}{18}-\frac{3}{2} \cos x+\frac{3}{10} \cos 2 x-\frac{17}{90} \cos 3 x=\frac{43}{3360} x^{8}-\frac{101}{18,900} x^{10}+\frac{47,177}{39,916,800} x^{12}+O\left(x^{14}\right) .
$$

\section{Main Results}

In the first part of this section we will prove our main results obtained using Padé approximant method for $\frac{\sqrt{\pi}}{2} \operatorname{erf}(x)$.

Theorem 1. The following inequalities hold:

(i) $\operatorname{erf}(x) \geq \frac{2}{\sqrt{\pi}} \cdot A(x)$ for all $x \geq 0$;

(ii) $\operatorname{erf}(x) \leq \frac{2}{\sqrt{\pi}} \cdot B(x)$ for all $x \in[0,4.418)$.

Proof. (i) The function $f(x)=\frac{\sqrt{\pi}}{2} \operatorname{erf}(x)-A(x)$ has the derivative

$$
f^{\prime}(x)=e^{-x^{2}}+\frac{\left(110 x^{3}+210 x\right)\left(156 x^{3}+360 x\right)}{\left(39 x^{4}+180 x^{2}+210\right)^{2}}-\frac{330 x^{2}+210}{39 x^{4}+180 x^{2}+210} .
$$

$f^{\prime}(x)$ is never zero when $x>0$, therefore $h(x)$ has no critical points for $x \geq 0$.

$f(x)$ has a global minimum at $x=0$. Since $f(0)=0$ it follows that $\operatorname{erf}(x) \geq \frac{2}{\sqrt{\pi}} \cdot A(x)$ for all $x \geq 0$.

(ii) We consider the function $g(x)=\frac{\sqrt{\pi}}{2} \operatorname{erf}(x)-B(x)$.

In order to find all positive critical points of $g$, first we compute $g^{\prime}(x)$.

$$
\begin{aligned}
g^{\prime}(x)= & e^{-x^{2}}+\frac{113,400 x\left(232 x^{7}-3960 x^{5}+5040 x^{3}+75,600 x\right)}{\left(29 x^{8}-660 x^{6}+1260 x^{4}+37,800 x^{2}+113,400\right)^{2}}- \\
& -\frac{113,400}{29 x^{8}-660 x^{6}+1260 x^{4}+37,800 x^{2}+113,400} .
\end{aligned}
$$

Solving the equation $g^{\prime}(x)=0$ yields $x \approx 3.77096$.

Now we evaluate $g(x)$ at the critical points and the endpoints of the domain, taking limit at the $\infty$ :

$$
g(0)=0, g(3.77096)=-1.32154, \lim _{x \rightarrow \infty} g(x)=\frac{\sqrt{\pi}}{2} .
$$


The function $\mathrm{g}$ has only one $\operatorname{root} x \approx 4.418$.

Therefore, $g(x) \leq 0$ for all $x \in[0,4.418]$ or, equivalently, $\operatorname{erf}(x) \leq \frac{2}{\sqrt{\pi}} \cdot B(x)$ for all $x \in[0,4.418]$.

Since $\operatorname{erf}(4.418)=0.999999999584246$, the error function can be considered $\operatorname{erf}(x)=1$ for $x \in$ $[4.418, \infty)$, hence our rational approximation offer good bounds near the origin for the error function.

The proof of Theorem 1 is complete.

In the following we will prove that our rational inequalities are more precise than Neuman's type inequalities.

Proposition 1. (i) For every $x \geq 0$, the inequality

$$
\frac{2}{\sqrt{\pi}} A(x) \geq \frac{2 x}{\sqrt{\pi}} \frac{e^{-x^{2}}+5 e^{-\frac{x^{2}}{5}}}{6}
$$

holds.

(ii) For every $x \geq 0$, the inequality

$$
\frac{2}{\sqrt{\pi}} B(x) \leq \frac{2 x}{\sqrt{\pi}} \frac{e^{-x^{2}}+2}{3}
$$

holds.

(iii) For every $x \in[0,3.10394] \cup[4.04171, \infty)$ the inequality

$$
\frac{2}{\sqrt{\pi}} B(x) \leq \frac{2 x}{\sqrt{\pi}} \frac{5 e^{-\frac{3 x^{2}}{5}}+4}{9}
$$

holds.

Proof. (i) The inequality from (i) takes the equivalent form:

$$
220 x^{2} e^{x 2}+420 e^{x^{2}}-1-5 e^{\frac{4 x^{2}}{5}}>0
$$

for all $x>0$.

It is sufficient to prove that

$$
420 e^{x^{2}}-1-5 e^{\frac{4 x^{2}}{5}}>0
$$

for all $x>0$, or, equivalently,

$$
\left(e^{x^{2}}-1\right)+e^{\frac{4 x^{2}}{5}}\left(419 e^{\frac{x^{2}}{5}}-5\right)>0
$$

for all $x>0$.

The above inequality is obviously true.

(ii) We consider the function

$$
h(x)=\frac{113,400}{29 x^{8}-660 x^{6}+1260 x^{4}+37,800 x^{2}+113,400}-\frac{1}{3}\left(e^{-x^{2}}+2\right) .
$$

To find all positive critical points, first we compute $h^{\prime}(x)$ :

$$
h^{\prime}(x)=\frac{2}{3} e^{-x^{2}} \cdot x-\frac{113,400\left(232 x^{7}-3960 x^{5}+5040 x^{3}+75,600 x\right)}{\left(29 x^{8}-660 x^{6}+1260 x^{4}+37,800 x^{2}+113,400\right)^{2}} .
$$


Solving the equation $h^{\prime}(x)=0$ yields the positive roots: $x=0, x \approx 2.59797, x \approx 3.71138$.

Moreover, the function $h^{\prime}(x)$ exists everywhere. Evaluate $h(x)$ at the critical points and take the limit:

$$
\begin{aligned}
h(0) & =0, h(2.59797)=-0.266605, \\
h(3.71138) & =-0.0767078 \text { and } \lim _{x \rightarrow \infty} h(x)=-\frac{2}{3} .
\end{aligned}
$$

Summarizing the results, we deduce that $h(x) \leq 0$ for all $x \geq 0$.

(iii) We introduce the function

$$
p(x)=\frac{113,400}{29 x^{8}-660 x^{6}+1260 x^{4}+37,800 x^{2}+113,400}-\frac{5 e^{-\frac{3 x^{2}}{9}}+4}{9} .
$$

In order to find all positive critical points, first we compute $p^{\prime}(x)$ :

$$
p^{\prime}(x)=\frac{2}{3} e^{-\frac{3 x^{2}}{5}} \cdot x-\frac{113,400\left(232 x^{7}-3960 x^{5}+5040 x^{3}+75,600 x\right)}{\left(29 x^{8}-660 x^{6}+1260 x^{4}+37,800 x^{2}+113,400\right)^{2}} .
$$

The equation $p^{\prime}(x)=0$ has the positive roots: $x=0, x \approx 2.4639, x \approx 3.71165$.

Evaluate $p(x)$ at the critical points and take the limit:

$$
\begin{aligned}
p(0) & =0, p(2.4639)=-0.0554863, \\
p(3.71165) & =0.145372 \text { and } \lim _{x \rightarrow \infty} p(x)=-\frac{4}{9} .
\end{aligned}
$$

The equation $p(x)=0$ has the positive roots: $x \approx 3.10394$ and $x \approx 4.04171$.

Summarizing the results, we obtain that $p(x) \leq 0$ for $x \in[0,3.10394] \cup[4.04171, \infty)$.

This completes the proof.

Therefore we improved the Neuman inequalities for all $x \geq 0$. We also refined the left-hand side of Yang-Chu's inequality for all $x \geq 0$ and the right-hand side of Yang-Chu's inequality for $x \in[0,3.10394] \cup[4.04171,4.418]$.

Our upper bound for the error function $\operatorname{erf}(x)$ also offers a better approximation near the origin than Pólya's inequality, due to the following proposition.

Proposition 2. The inequality

$$
\frac{2}{\sqrt{\pi}} B(x) \leq \sqrt{1-e^{-\frac{4 x^{2}}{\pi}}}
$$

holds for all $x \in[0,1.573]$.

Proof. The above inequality can be rewritten as

$$
\pi\left(e^{\frac{4 x^{2}}{\pi}}-1\right)\left(29 x^{8}-660 x^{6}+1260 x^{4}+37,800 x^{2}+113,400\right)^{2}-4(113,400 x)^{2} e^{\frac{4 x^{2}}{\pi}} \geq 0 .
$$

The function from the left-hand side has the positive roots: $x=0, x \approx 1.573, x \approx 4.37287$, therefore the function takes positive values for $x \in[0,1.573]$.

The proof is complete. 
In order to refine Komatsu's inequalities, first we use the change of variables $t=y \sqrt{2}$ in the integral $\int_{x}^{\infty} e^{-\frac{t^{2}}{2}} d t$, then we substitute $\frac{x}{\sqrt{2}}$ by $x$ in Komatsu's inequalities and we obtain Komatsu's inequalities for the complementary error function $\operatorname{erfc}(x)$ :

$$
\frac{2 e^{-x^{2}}}{x+\sqrt{x^{2}+2}}<\frac{\sqrt{\pi}}{2} \operatorname{erfc}(x)<\frac{e^{-x^{2}}}{x+\sqrt{x^{2}+1}}
$$

hold for all $x>0$ or, equivalent:

$$
1-\frac{2}{\sqrt{\pi}} \frac{e^{-x^{2}}}{x+\sqrt{x^{2}+1}}<\operatorname{erf}(x)<1-\frac{2}{\sqrt{\pi}} \frac{e^{-x^{2}}}{x+\sqrt{x^{2}+2}}
$$

hold for all $x>0$.

Using the Padé approximation for erf $f(x)$, we obtain an improved version of Komatsu's inequalities as follows.

Proposition 3. The following inequalities hold:
(i) $\frac{2}{\sqrt{\pi}} A(x) \geq 1-\frac{2}{\sqrt{\pi}} \frac{e^{-x^{2}}}{x+\sqrt{x^{2}+1}}$ for all $x \in[0,1.28089)$,
(ii) $\frac{2}{\sqrt{\pi}} B(x) \leq 1-\frac{2}{\sqrt{\pi}} \frac{e^{-x^{2}}}{x+\sqrt{x^{2}+2}}$ for all $x \in[0,1.33740041) \subset[0,4.418)$.

Proof. (i) The inequality from (i) takes the equivalent form:

$$
\begin{gathered}
e^{x^{2}}\left(-\frac{39 \sqrt{\pi}}{2} x^{4}+110 x^{3}-90 \sqrt{\pi} x^{2}+210 x-105 \sqrt{\pi}\right)+ \\
+\left(\sqrt{x^{2}+1}-x\right)\left(39 x^{4}+180 x^{2}+210\right)>0 .
\end{gathered}
$$

The function from the left-hand side has only one positive root $x \approx 1.28089$ and it is positive for $x \in(0,1.28089)$.

(ii) The inequality from (ii) can be rewritten as follows:

$$
\begin{gathered}
e^{x^{2}}\left(-\frac{29 \sqrt{\pi}}{2} x^{8}+330 \sqrt{\pi} x^{6}-630 \sqrt{\pi} x^{4}-18,900 \sqrt{\pi} x^{2}+113,400 x-56,700 \sqrt{\pi}\right)+ \\
+\left(\sqrt{x^{2}+2}-x\right)\left(\frac{29}{2} x^{8}-330 x^{6}+630 x^{4}+18,900 x^{2}+56,700\right)>0 .
\end{gathered}
$$

The function from the left-hand side has two positive roots: $x \approx 1.33740$ and $x \approx 4.37287$ and it is positive for $x \in(0,1.33740) \cup(4.37287, \infty)$.

Therefore, we improve Komatsu's inequalities near the origin providing sharp bounds of rational types for the error function.

In the following we derive approximation to four decimals of precision for the function $\frac{\sqrt{\pi}}{2} \operatorname{erf}(x)$ on a large neighbourhood of the origin.

Theorem 2. The approximation $\frac{\sqrt{\pi}}{2} \operatorname{erf}(x) \simeq C(x)$ is valid for all $x,|x| \leq 2.4$, with absolute error $|\varepsilon(x)|<$ $8.22775 \times 10^{-4}$.

Proof. The function

$$
\varepsilon(x)=\frac{\sqrt{\pi}}{2} \operatorname{erf}(x)-C(x)
$$


has the derivative

$$
\varepsilon^{\prime}(x)=e^{-x^{2}}+\frac{21}{5} \cdot \frac{E(x)}{F(x)}
$$

where

$$
\begin{aligned}
E(x)= & 151,463,081 x^{10}-640,919,835 x^{8}-5,296,822,800 x^{6}+ \\
& +31,069,085,040 x^{4}+132,620,407,920 x^{2}-999,139,291,920
\end{aligned}
$$

and

$$
F(x)=\left(10,519 x^{6}+151,725 x^{4}+888,300 x^{2}+2,048,508\right)^{2} .
$$

The equation $\varepsilon^{\prime}(x)=0$ has the roots $x_{1} \simeq-1.7103, x_{2} \simeq 0, x_{3} \simeq 1.7103$.

Partition the domain into intervals with endpoints at the critical points:

$$
(-\infty,-1.7103),(-1.7103,0),(0,1.7103) \text { and }(1.7103, \infty) .
$$

Then we have $\varepsilon^{\prime}(x)>0$ on $(-\infty,-1.7103)$ and $(1.7103, \infty)$ respectively and $\varepsilon^{\prime}(x)<0$ on $(-1.7103,1.7103)$.

On the interval $(-\infty,-1.7103)$, the function $\varepsilon$ is increasing from $\lim _{x \rightarrow-\infty} \varepsilon(x)=-\frac{\sqrt{\pi}}{2} \simeq$ -0.886227 to $\varepsilon(-1.7103)=1.95966 \times 10^{-5}$.

The root $x_{2}=0$ is a critical point, but it is not an extreme point of the function $\varepsilon$, therefore on the interval $(-1.7103,1.7103)$, the absolute error function $\varepsilon$ is decreasing from $\varepsilon(-1.7103)=$ $1.95966 \times 10^{-5}$ to $\varepsilon(1.7103)=-1.95966 \times 10^{-5}$.

On the interval $(1.7103, \infty)$, the function $\varepsilon$ is increasing from $\varepsilon(1.7103)=-1.95966 \times 10^{-5}$ to $\lim _{x \rightarrow \infty} \varepsilon(x)=\frac{\sqrt{\pi}}{2} \simeq 0.886227$.

Since we have $\varepsilon(2.4)=8.22775 \times 10^{-4}$ and $\varepsilon(2.5)=1.2898 \times 10^{-3}$, we consider our a approximation on $[0,2.4]$. For negative $x$, one can use the identities $\operatorname{erf}(-x)=-\operatorname{erf}(x)$ and $C(-x)=-C(x)$, therefore we extend our approximation on $[-2.4,2.4]$.

The proof of Theorem 2 is complete.

Remark 1. We find the approximation $\frac{\sqrt{\pi}}{2} \operatorname{erf}(x) \simeq C(x)$ with the absolute error $|\mathcal{E}(x)|<8.22775 \times 10^{-4}$. Therefore we propose the following approximation for the error function er $f(x)$ :

$$
\eta(x)=\left\{\begin{array}{c}
\frac{2}{\sqrt{\pi}} C(x) ;-2.4 \leq x \leq 2.4 \\
1 ; \text { otherwise }
\end{array}\right.
$$

where the absolute error for the first form is $\left|\varepsilon_{1}(x)\right|<\frac{2}{\sqrt{\pi}} \times 8.22775 \times 10^{-4}=9.28402 \times 10^{-4}$ and the absolute error for the second form is $\left|\varepsilon_{2}(x)\right|<|1-\operatorname{erf}(2.4)|=6.88514 \times 10^{-4}$.

In the second part of this section we will establish our approximations of the error function using Fourier trigonometric series expansions.

Theorem 3. The following inequalities

$$
-\frac{7}{18} x+\frac{3}{2} \sin x-\frac{3}{20} \sin 2 x+\frac{17}{270} \sin 3 x<\frac{\sqrt{\pi}}{2} \operatorname{erf}(x)<\frac{3}{2} x-\frac{4}{3} \sin x+\frac{5}{12} \sin 2 x
$$

hold for all $x>0$. 
Proof. In order to prove the right - hand side inequality, we consider the function $f_{1}:(0, \infty) \rightarrow \mathbb{R}$, $f_{1}(x)=\frac{\sqrt{\pi}}{2} \operatorname{erf}(x)-\frac{3}{2} x+\frac{4}{3} \sin x-\frac{5}{12} \sin 2 x$.

The derivative of the function $f_{1}$ is

$$
f_{1}^{\prime}(x)=e^{-x^{2}}-\frac{3}{2}+\frac{4}{3} \cos x-\frac{5}{6} \cos 2 x .
$$

We intend to show that $f_{1}^{\prime}<0$ on $(0, \infty)$ or, equivalently,

$$
e^{-x^{2}}<-\frac{4}{3} \cos x+\frac{5}{6} \cos 2 x+\frac{3}{2}, \text { for all } x \in(0, \infty) .
$$

Since

$$
-\frac{4}{3} \cos x+\frac{5}{6} \cos 2 x+\frac{3}{2}=\frac{5 \cos ^{2} x-4 \cos x+2}{3}>0
$$

for all $x \in(0, \infty)$, we can log the above inequality and it remains to be proved that

$$
-x^{2}<\log \left(-\frac{4}{3} \cos x+\frac{5}{6} \cos 2 x+\frac{3}{2}\right),
$$

for all $x \in(0, \infty)$.

The function $f_{2}:(0, \infty) \rightarrow \mathbb{R}, f_{2}(x)=-x^{2}-\log \left(-\frac{4}{3} \cos x+\frac{5}{6} \cos 2 x+\frac{3}{2}\right)$ has the derivatives

$$
f_{2}^{\prime}(x)=\frac{2(4 \sin x-5 \sin 2 x)}{8 \cos x-5 \cos 2 x-9}-2 x
$$

and

$$
f_{2}^{(2)}(x)=\frac{-32 \sin ^{4}\left(\frac{x}{2}\right)\left((5 \cos x+2)^{2}+2\right)}{(-8 \cos x+5 \cos 2 x+9)^{2}}<0
$$

for all $x>0$.

Then $f_{2}^{\prime}$ is strictly decreasing on $(0, \infty)$. As $f_{2}^{\prime}(0)=0$, we get $f_{2}^{\prime}<0$ on $(0, \infty)$.

Continuing the algorithm, finally we find $f_{2}<0$ on $(0, \infty)$.

In order to prove the left-hand side inequality, first we notive that the function

$$
f_{3}(x)=-\frac{7}{18} x+\frac{3}{2} \sin x-\frac{3}{20} \sin 2 x+\frac{17}{270} \sin 3 x
$$

has the positive roots $x=0, x \approx 2.57617$.

Since $f_{3}\left(\frac{\pi}{2}\right)>0$, it follows that $f_{3}>0$ only on $(0,2.57617)$, hence we have to show the left-hand side inequality only for $x \in(0,2.57617)$.

We introduce the function $f_{4}:(0,2.57617) \rightarrow \mathbb{R}, f_{4}(x)=\frac{\sqrt{\pi}}{2} \operatorname{erf}(x)+\frac{7}{18} x-\frac{3}{2} \sin x+\frac{3}{20} \sin 2 x-$ $\frac{17}{270} \sin 3 x$.

The derivative of the function $f_{4}$ is

$$
f_{4}^{\prime}(x)=e^{-x^{2}}-\frac{3}{2} \cos x+\frac{3}{10} \cos 2 x-\frac{17}{90} \cos 3 x+\frac{7}{18} .
$$

We have to prove that $f_{4}^{\prime}>0$ on $(0,2.57617)$, or equivalently,

$$
e^{-x^{2}}>\frac{3}{2} \cos x-\frac{3}{10} \cos 2 x+\frac{17}{90} \cos 3 x-\frac{7}{18} .
$$

The function $f_{5}(x)=\frac{3}{2} \cos x-\frac{3}{10} \cos 2 x+\frac{17}{90} \cos 3 x-\frac{7}{18}$ has on $(0,2.57617)$ only the root $x \approx$ 1.46966. Since $f_{5}\left(\frac{\pi}{2}\right)<0$, then $f_{5}>0$ only on $(0,1.46966)$.

Hence it remains to verify the inequality (3) only for $x \in(0,1.46966) \subset\left(0, \frac{\pi}{2}\right)$. 
We log the inequality (3) and we obtain

$$
-x^{2}-\log \left(\frac{3}{2} \cos x-\frac{3}{10} \cos 2 x+\frac{17}{90} \cos 3 x-\frac{7}{18}\right)>0
$$

on $(0,1.46966)$.

We consider the function $f_{6}:(0,1.46966) \rightarrow \mathbb{R}, f_{6}(x)=-x^{2}-\log \left(\frac{3}{2} \cos x-\frac{3}{10} \cos 2 x\right.$ $\left.+\frac{17}{90} \cos 3 x-\frac{7}{18}\right)$.

Its derivatives are

$$
f_{6}^{\prime}(x)=-2 x+\frac{3(45 \sin x-18 \sin 2 x+17 \sin 3 x)}{-35+135 \cos x-27 \cos 2 x+17 \cos 3 x}
$$

and

$$
f_{6}^{(2)}(x)=\frac{32 \sin ^{6}\left(\frac{x}{2}\right)\left(2312 \cos ^{3} x+4182 \cos ^{2} x+3600 \cos ^{2} x+1516\right)}{(-135 \cos x+27 \cos 2 x-17 \cos 3 x+35)^{2}} .
$$

Evidently, $f_{6}^{(2)}(x)>0$ on $\left(0, \frac{\pi}{2}\right) \supset(0,1.46966)$.

Then $f_{6}^{\prime}$ is strictly increasing on $(0,1.46966)$. As $f_{6}^{\prime}(0)=0$, we find that $f_{6}^{\prime}>0$ on $(0,1.46966)$.

Finally, we have $f_{6}>0$ on $(0,1.46966)$.

The proof of the Theorem 3 is complete.

In the following we derive approximation to three decimals of precision for the function $\operatorname{erf}(x)$ on a large neighbourhood of the origin.

We note by

$$
G(x)=\frac{2}{\sqrt{\pi}}\left(-\frac{7}{18} x+\frac{3}{2} \sin x-\frac{3}{20} \sin 2 x+\frac{17}{270} \sin 3 x\right)
$$

and

$$
H(x)=\frac{2}{\sqrt{\pi}}\left(\frac{3}{2} x-\frac{4}{3} \sin x+\frac{5}{12} \sin 2 x\right) .
$$

In Theorem 3 we proved that

$$
G(x)<\operatorname{erf}(x)<H(x)
$$

for all $x>0$.

Now we consider the convex combination

$$
\theta(x)=\operatorname{erf}(x)-0.77 G(x)-(1-0.77) H(x)
$$

or, equivalently,

$$
\theta(x)=\operatorname{erf}(x)-0.051403 x-0.957242 \sin x+0.022191 \sin 2 x-0.054705 \sin 3 x .
$$

The derivative of $\theta(x)$ is

$$
\theta^{\prime}(x)=1.12838\left(e^{-x^{2}}-0.848334 \cos x+0.0399333 \cos 2 x-0.145444 \cos 3 x-0.045555\right) .
$$

The roots of the function $\theta^{\prime}$ on the interval $[0,1.933]$ are $x=0$ and $x \approx 1.571371$. Since $\frac{\pi}{2} \in$ $(0,1.571371)$ and $\theta^{\prime}\left(\frac{\pi}{2}\right)<0$, it follows that $\theta^{\prime}<0$ on $(0,1.571371)$ and $\theta^{\prime}>0$ on $(1.571371,1.933)$, hence $\theta$ is strictly decreasing on $(0,1.571371)$ and $\theta$ is strictly increasing on $(1.571371,1.933)$.

Then $\min _{0 \leq x \leq 1.933} \theta(x) \approx-0.009602$ at $x \approx 1.571371, \max _{0 \leq x \leq 1.933} \theta(x) \approx 0.009997$ at $x \approx 1.933$ and $\theta(0)=0$. 
Therefore we find the approximation $\operatorname{erf}(x) \approx J(x)$, where $J(x)=0.051403 x+0.957242 \sin x-$ $0.022191 \sin 2 x+0.054705 \sin 3 x$ with the absolute error $|\theta(x)| \leq 9.99747 \times 10^{-3}$.

Since $|1-\operatorname{erf}(1.933)|=6.26319 \times 10^{-3}$, we propose the 3 following approximation to three decimals of precision for the error function $\operatorname{erf}(x)$ :

$$
\varphi(x)=\left\{\begin{array}{l}
J(x) ; 0 \leq x \leq 1.933 \\
1 ; \text { otherwise }
\end{array}\right.
$$

\section{Application Case}

As an example to apply the error function, one case is considered for the diffusion and reactions in sediments.

We assume that oxygenated water comes in contact with sediments at the bottom of a lake at the time of "fall turnover". The water contains $300 \mu$ moles of $\mathrm{O}_{2} /$ liter. We suppose that no reactions consume the oxygen. We want to find what is the concentration of oxygen in sediments after one day, one week and one month after the turnover event.

Mathematically, the diffusion problem for a constant source region diffusing into a space of unlimited extent ("semi-infinite half-space") is to solve

$$
\frac{\partial C}{\partial t}=D \cdot \frac{\partial^{2} C}{\partial x^{2}}
$$

given the boundary conditions that the concentration at the sediment-water interface $C_{0}$ is always fixed and no reactions consume the oxygen, and initial condition that the concentration below the boundary is initially everywhere 0 .

This linear PDE, having a solution that requires one initial condition and two boundary conditions is called Fick's second law for isotropic one-dimensional diffusion with $D$ independent of concentration.

The solution to this problem involves the error function:

$$
C(x, t)=C_{0} \operatorname{erfc}\left(\frac{x}{2 \sqrt{D t}}\right)
$$

where $C(x, t)$ is the concentration at a given depth and time, $C_{0}$ is the concentration at the interface, $D$ is the diffusion coefficient, $x$ is depth below the interface, and $t$ is time.

An appropriate value of the diffusion constant $D$ is $2 \times 10^{-5} \mathrm{~cm}^{2} \mathrm{~s}^{-1}$. We choose a depth range $x$ of $0-20 \mathrm{~cm}$. Since the units of time in the diffusion constant are seconds, we calculate our valued for concentration using seconds for $t$ to obtain a good result.

In order to solve this equation, we will use our approximation for the error function:

$$
\begin{aligned}
C(x, t) & =C_{0}\left(1-\operatorname{erf}\left(\frac{x}{2 \sqrt{D t}}\right)\right) \\
& =C_{0}\left(1-\eta\left(\frac{x}{2 \sqrt{D t}}\right)\right),
\end{aligned}
$$

where $\eta(x)$ is defined by (2) or $C(x, t)=C_{0}\left(1-\varphi\left(\frac{x}{2 \sqrt{D t}}\right)\right)$, where $\varphi(x)$ is defined by (4).

\section{Conclusions}

In our work we use Padé approximation method and Fourier series method in order to obtain approximations for the error function. Using Padé approximation method, we obtain the following bounds: $\operatorname{erf}(x) \geq \frac{2}{\sqrt{\pi}} A(x)$, for all $x \geq 0$ and $\operatorname{erf}(x) \leq \frac{2}{\sqrt{\pi}} B(x)$, for all $x \in[0,4.418)$ and the following 
approximation to four decimals of precision for the function $\operatorname{erf}(x)$ on a large neighbourhood of the origin:

$$
\eta(x)=\left\{\begin{array}{c}
\frac{2}{\sqrt{\pi}} C(x) ;-2.4 \leq x \leq 2.4 \\
1 ; \text { otherwise }
\end{array}\right.
$$

with the absolute error $\left|\varepsilon_{1}(x)\right|<9.28402 \times 10^{-4}$, where $C(x)=\frac{302,379 x^{5}+1,027,320 x^{3}+10,242,540 x}{52,595 x^{6}+758,625 x^{4}+4,441,500 x^{2}+10,242,540}$. Furthermore, using Fourier series method, we obtain the following bounds:

$$
G(x)<\operatorname{erf}(x)<H(x) \text {, for all } x>0
$$

and the following approximation to three decimals of precision for the function $\operatorname{erf}(x)$ on a large neighbourhood of the origin:

$$
\varphi(x)=\left\{\begin{array}{l}
J(x) ; 0 \leq x \leq 1.933 \\
1 ; \text { otherwise }
\end{array}\right.
$$

with the absolute error $|\theta(x)| \leq 9.99747 \times 10^{-3}$, where $J(x)=0.051403 x+0.957242 \sin x-$ $0.022191 \sin 2 x+0.054705 \sin 3 x$.

The results are simple expressions which have acceptable deviations in connection with engineering calculations. The absolute error for the error functions are also comparable to other approximations found in the literature. The approximate error functions can be employed on the solution of practical problems like the one related to diffusion and reactions in sediments.

Funding: The APC was funded by “Dunărea de Jos" University of Galaţi, Romania.

Conflicts of Interest: The author declares no conflict of interest.

\section{References}

1. Abramowitz, M.; Stegun, I.A. Handbook of Mathematical Function with Formulas, Graphs and Mathematical Tables; National Bureaus of Standards: Gaithersburg, MA, USA, 1964.

2. Burr, I.W. A useful approximation to the normal distribution function, with applications to simulation. Technometrics 1967, 9, 647-651. [CrossRef]

3. Crank, J. The Mathematics of Diffusion; Clarendon Press: Oxford, UK, 1975.

4. Chu, J.-T. On bounds for the normal integral. Biometrika 1955, 42, 263-265. [CrossRef]

5. Hastings, C. Approximations for Digital Computers; Princeton University Press: Princeton, NJ, USA, 1955; pp. 167-185.

6. Karlsson, H.T.; Bjerle, I. A simple approximation of the error function. Comput. Chem. Eng. 1980, 4, 67-68. [CrossRef]

7. Winitzki, S. Uniform approximations for transcendental functions. In Proc. ICCSA-2003; LNCS 2667/2003, 962; Springer: Berlin/Heidelberg, Germany, 2003.

8. Pólya, G. Remarks on computing the probability integral in one and two dimensions. In Proceeding of the Berkeley Symposium on Mathematical Statistics and Probability; University of California Press: Berkeley, CA, USA, 1949.

9. Vasquez-Leal, H.; Castaneda-Sheissa, R. High Accurate Simple Approximation of Normal Distribution. Math. Probl. Eng. 2012. [CrossRef]

10. Yang, Z.-H.; Chu, Y.-M. On approximating the error function. J. Inequal. Appl. 2016, 2016, 311. [CrossRef]

11. Yerukala, R.; Boiroju, N.K. Approximations to Standard Normal Distribution Function. Int. J. Eng. Sci. Eng. Res. 2015, 6, 515-518.

12. Norton, R.M. Pocket-Calculator Approximation for Area under the Standard Normal Curve. Am. Stat. 1989, 43, 24-26.

13. Yang, Z.-H.; Qian, W.-M.; Chu, Y.-M.; Zhang, W. On approximating the error function. Math. Inequal. Appl. 2018, 21, 469-479. [CrossRef]

14. Qi, F.; Cui, L.-H.; Xu, S.-L. Some inequalities constructed by Tchebysheff's integral inequality. Math. Inequal. Appl. 1999, 2, 517-528. [CrossRef] 
15. Neuman, E. Inequalities and bounds for the incomplete gamma function. Result. Math. 2013, 3-4, 1209-1214. [CrossRef]

16. Ito, K.; Mc, Kean, H.P. Diffusion Processes and Their Sample Paths; Springer: Berlin/Heidelberg, Germany, $1965 ;$ p. 17.

17. Boyd, J.P. Chebyshev and Fourier Spectral Methods; Dover: New York, NY, USA, 2000.

18. Mitrinović, D.S. Analytic Inequalities; Springer: Berlin/Heidelberg, Germany, 1970.

19. Lutovac, T.; Malešević, B.; Mortici, C. The natural algorithmic approach of mixed trigonometric-Polynomial problems. J. Inequal. Appl. 2017, 2017, 116. [CrossRef] [PubMed]

20. Nenezić, M.; Maleševixcx, B.; Mortici, C. Accurate approximations of some expressions involving trigonometric functions. Appl. Math. Comput. 2016, 283, 299-315.

Publisher's Note: MDPI stays neutral with regard to jurisdictional claims in published maps and institutional affiliations.

(C) 2020 by the author. Licensee MDPI, Basel, Switzerland. This article is an open access article distributed under the terms and conditions of the Creative Commons Attribution (CC BY) license (http:/ / creativecommons.org/licenses/by/4.0/). 